\title{
Enfermedad de Still, un diagnóstico diferencial importante: Reporte de un Caso
}

Still's disease, an important differential diagnosis: Report of a Case

Catalina Quilindo ${ }^{1}$

Kimberly Morales ${ }^{1}$

Andrés Guerrero ${ }^{2}$

Recibido: $15 / 02 / 2017$
Revisado: $13 / 03 / 2017$
Aceptado: $14 / 06 / 2017$

\section{Resumen}

La enfermedad de Still es un transtorno inflamatorio multisistémico de causa desconocida caracterizada por episodios de fiebre, rash cutáneo, artralgia y principalmente mialgias. Este es un caso de una paciente de 24 años quien presentó un cuadro febril subagudo asociado a artralgias, cefalea, lesiones maculopapilares cutáneas, adenomegalias cervicales, submandibulares, axilares e inguinales. Los estudios iniciales reportaron elevación de reactantes de fase aguda, ferritina sérica, disfunción hepática y perfil inmune negativo. La biopsia de piel reportó signos compatibles con eritema multiforme. Fue considerado inicialmente múltiples diagnósticos diferenciales de características infecciosas y autoinmunes. Se indicó manejo esteroideo y sintomático. La paciente se recuperó y fue dada de alta sin complicaciones. Se discute el síndrome de Still como una enfermedad rara que genera aún un reto diagnóstico para el clínico.

Palabras clave: Enfermedad de Still, artralgia, exantema, ferritina, factor reumatoide.

\begin{abstract}
Still's disease is a multisystemic inflammatory disorder of unknown cause characterized by episodes of fever, skin rash, arthralgia and mainly myalgias. This is a case of a 24 -year-old patient who presented a subacute febrile episode associated with arthralgia, headache, cutaneous maculopapillary lesions, cervical, submandibular, axillary and inguinal lymph nodes. Initial studies reported elevation of acute phase reactants, serum ferritin, liver dysfunction and negative immune profile. The skin biopsy reported signs compatible with erythema multiforme. Initially, multiple differential diagnoses of infectious and autoimmune characteristics were considered. Steroid and symptomatic management was indicated. The patient recovered and was discharged without complications. Still syndrome is discussed as a rare disease that still generates a diagnostic challenge for the clinician.
\end{abstract}

Key words: Still disease, arthralgia, rash, ferritin, rheumatoid factor.

\footnotetext{
${ }^{1}$ Médico y Cirujano. Universidad del Cauca.

${ }^{2}$ Médico residente de Medicina Interna. Universidad del Cauca. Facultad Ciencias de la Salud.

Contacto: catalinaqg1420@gmail.com
} 


\section{Introducción}

La enfermedad de Still es una rara afección multisistemica de características inflamatorias variables y etiología desconocida (1). Se considera la presencia de una distribución étnica y de género homogénea, con picos de aparición asociados a la edad de 15 a 25 y 36 a 46 años (2). El rastreo paraclínico generalmente evidencia un proceso inflamatorio sistémico, pero sin ningún hallazgo francamente específico. Se considera que es una condición de abordaje primordialmente clínico, en la cual se tiene en cuenta una amplia gama de diagnósticos diferenciales, dentro de los que se incluyen patología infecciosa, autoinmune y principalmente neoplásica (3). Existen diferentes grupos de criterios para el diagnóstico, entre ellos están: los criterios de Goldman, Calabro, Cush, Reginato. Sin embargo los criterios de Yamaguchi son actualmente los más importantes (4). El manejo, inicialmente está basado en uso de anti-inflamatorios no esteroideos y glucocorticoides, requiriendo en casos más complejos medicamentos inmunomoduladores. (5).

\section{Descripción del Caso}

Paciente femenina de 24 años de edad. Sin antecedentes médicos previos. Presenta cuadro clínico de 15 días de evolución previos a ingreso hospitalario caracterizado por aparición de artralgias, lumbalgia, cefalea y malestar general asociado a fiebre subjetiva. Posteriormente con aparición de lesiones cutáneas eritematosas en muslos, no pruriginosas. De forma ambulatoria fue manejada con gentamicina, doxiciclina, naproxeno y azitromicina considerándose la presencia de un tipo de patología infecciosa. A pesar de ello la paciente no presenta mejoría por lo que consulta al servicio de urgencias. Fue hospitalizada por persistencia del cuadro clínico descrito. Al ingreso se documenta aumento del eritema maculopapular en muslos y abdomen; se identifica al examen físico exhaustivo la presencia de eritema amigdalar, lesión herpetiforme en labio inferior y adenomegalias no dolorosas a nivel cervical, submandibular, axilar e inguinal. Durante su internación se evidenció en los paraclínicos (tabla 1 y 2) la presencia de respuesta inflamatoria sistémica dada por leucocitosis, proteína c reactiva $(\mathrm{PCR}=10,1)$ y velocidad de sedimentación globural $(\mathrm{VSG}=98 \mathrm{ng} / \mathrm{ml}$ ) evadas. Además se evidenció compromiso hepático con elevación de transaminasas Glutamano Oxalacetato Transaminasa (TGO $=225 \mathrm{UI} / \mathrm{ml}$ ) y el Glutamano Piruvato Transaminasa $(\mathrm{TGP}=230 \mathrm{UI} / \mathrm{ml})$. Posteriormente, se descarta infección aguda activa; el perfil inmunológico fue reportado como normal y ante la posterior sospecha clínica de una enfermedad de Still del adulto se solicitaron valores de ferritina sérica, evidenciando niveles elevados $(2.000 \mathrm{ng} / \mathrm{ml})$. Finalmente se realizó biopsia de piel la cual reportó hallazgos sugestivos de eritema multiforme Frente al contexto clínico descrito se decide iniciar manejo con esteroide endovenoso, evidenciando adecuada respuesta; progresiva reducción de marcadores clínicos y paraclínicos de respuesta inflamatoria, desaparición de rash y normalización de función hepática. La paciente fue egresada sin complicación alguna y sin recurrencia al momento de realización del presente texto.

Table 1. Resultado de paraclínicos.

\begin{tabular}{l|c}
\multicolumn{1}{c|}{ PARACLÍNICOS } & \\
\hline LDH & $1092 \mathrm{UI} / \mathrm{ml}$ \\
\hline FOSFATASA ALCALINA & 81 \\
\hline COMPLEMENTO C3 Y C4 & Normales \\
\hline FACTOR REUMATOIDEO & Negativo \\
\hline IGG /IGM DENGUE & Negativo \\
\hline IGG /IGMEBSTEINBARR & Negativo \\
\hline IGM TOXOPLASMA & Negativo \\
\hline VIH & Negativo \\
\hline
\end{tabular}

Table 2. Resultados de Paraclínicos.

\begin{tabular}{l|c}
\multicolumn{1}{c}{ PARACLÍNICOS } & VALOR \\
\hline LEUCOCITOS & $9800 \mathrm{~mm} 3$ \\
\hline NEUTROFILOS & $8700 \mathrm{~mm} 3$ \\
\hline HEMOGLOBINA & 1 \\
\hline HEMATOCRITO & 1 \\
\hline PLAQUETAS & 156200 \\
\hline PCR & 10,1 \\
\hline VSG & $98 \mathrm{ng} / \mathrm{ml}$ \\
\hline FERRITINA & $2000 \mathrm{ng} / \mathrm{ml}$ \\
\hline GOT & $225 \mathrm{UI} / \mathrm{ml}$ \\
\hline TGP & $230 \mathrm{UI} / \mathrm{ml}$ \\
\hline
\end{tabular}

\section{Discusión}

La enfermedad de Still es una patología multisistémica inflamatoria de etiología desconocida hasta el momento (6). El nombre de la enfermedad perpetua en honor a su primera descripción en 1897 por el Dr. George Still, publicada en su monografía: "On a form of chronic joint disease in children". Actualmente dicha condición, se conoce como Artritis Idiopática Juvenil, pero en 1971, Bywaters acuña el término para describir adultos con una condición de características similares (7). Se considera como una entidad poco común, con incidencia reportada de 0.16 casos/100.000 personas/año (8). Se ha planteado la enfermedad de Still como un síndrome reactivo, en el que agentes infecciosos diversos (Epstein-Barr Virus, parvovirus B19, Citomagalovirus, Herpes Virus 6, VIH, Virus Coxsakie, Campylobacter jejuni, entre otros) pueden disparar la enfermedad en sujetos genéticamente susceptibles (9).

Para poder enfocar un diagnóstico preciso se requiere conocer las manifestaciones clínicas más comunes, dentro de las que se incluyen cuatro: artralgias (98 $100 \%$, fiebre mayor o igual a $39^{\circ} \mathrm{C}(83-100 \%)$, mialgias $(84-90 \%), \operatorname{rash}(87-90 \%)$ y odinofagia $(50$ - 92\%). Las artralgias comienzan concomitantemente con la fiebre, involucran cualquier articulación, pueden migrar inicialmente y se tornan más estables con el curso de la enfermedad (10). El rash se caracteriza por 
presentar discretas lesiones maculares o maculopapulares, no pruriginosas, color rosado salmón, las cuales son transitorias y principalmente visibles durante la fiebre (11). Algunos pacientes pueden presentar linfadenopatías (hasta un 65\%), hepatoesplenomegalia y/o serositis (12). Estas características se correlacionan estrechamente con el caso presentado en donde la paciente debutó con un síndrome febril de más de 15 días de evolución, con picos febriles cuantificados hasta los $39^{\circ} \mathrm{C}$ y que se acompañó de artralgias a nivel glenohumeral, de tobillo izquierdo y rodillas principalmente, odinofagia y la aparición del característico rash previamente descrito. Además, se reporta la presencia de adenopatías axilares izquierdas inicialmente y con posterior identificación a nivel cervical e inguinal, también descrito en la literatura aunque con una frecuencia de presentación menor (13). En ocasiones se puede llegar a requerir el conocimiento de manifestaciones atípicas de la enfermedad, dado que se han reportado casos asociados a efusión pleural eosinofílica (14), miopericarditis (15), meningitis aséptica (16) o incluso choque y falla orgánica múltiple (8), entre otros. No se encuentran características atípicas en el presente caso.

No existen hallazgos de laboratorio patognomónicos. Se describe la utilidad de pruebas que reflejan inflamación sistémica como la VSG y la PCR (17); en el presente caso se documenta ascenso inicial de la PCR, además de VSG elevada. El recuento leucocitario elevado (usualmente de 10.000 a 15.000 con más del $80 \%$ de granulocitos) (18) es también marcador descrito y se evidencia dentro de los controles realizados a la paciente. El factor reumatoideo y los anticuerpos antinucleares se describen característicamente como negativos, lo que ayuda a descartar algunas condiciones reumatológicas (19). Efectivamente el factor reumatoideo solicitado durante la estancia hospitalaria fue negativo, al igual que anticuerpos antinucleares y anticuerpos anti DNA. Además, se reporta una incidencia elevada de hiperferritinemia y se plantea que este marcador puede ser útil para el monitoreo de la enfermedad durante el tratamiento (20). En nuestro caso se evidencia cómo los valores de ferritina se elevaron significativamente hasta los $2.000 \mathrm{ng} / \mathrm{ml}$ con valor de referencia de laboratorio entre 6 y $159 \mathrm{ng} / \mathrm{ml}$. No se contó con seguimiento de este marcador. Se han planteado ocho grupos de criterios diagnósticos para esta condición, pero los más utilizados y validados son los criterios de Yamaguchi (21) (tabla 3), para los cuales se ha reportado una sensibilidad y especificidad del $96.2 \%$ y $92.1 \%$ respectivamente (10). $\mathrm{Al}$ aplicarlos al caso en cuestión se encuentra que la paciente presentaba todos los criterios mayores (fiebre de $39^{\circ} \mathrm{C}$ de más de 7 días de duración, artralgias de más de dos semanas de duración, rash típico, y leucocitosis) y menores descritos (odinofagia, linfadenopatía, disfunción hepática y factor reumatoide y anticuerpos antinucleares negativos).

Table 3. Criterios diagnósticos para la enfermedad de Still. (Yamaguchi, 1992).

\section{CRITERIOS MAYORES}

- Fiebre mayor de $39^{\circ} \mathrm{C}$, que dure 1 semana o más.

- Artralgia o artritis, que duren 2 o más semanas.

- Rash típico.

- Leucocitosis mayor de $10.000 \mathrm{cel} / \mathrm{mm} 3$ con más del $80 \%$ de polimorfonucleares.

\section{CRITERIOS MENORES}

- Odinofagia.

- Linfadenopatías y/o esplenomegalia.

- Anormalidad en pruebas de función hepática.

- Pruebas negativas para anticuerpos antinucleares y factor reumatoideo.

\section{CRITERIOS DE EXCLUSIÓN}

- Infección

- Malignidad

- Otras enfermedades reumáticas

* Se requieren cinco o más criterios, de los cuales dos deben ser mayores.

Un diagnóstico predominantemente clínico obliga a descartar condiciones infecciosas, neoplásicas y autoinmunes principalmente. Se plantea que las enfermedades que con más frecuencia deben descartarse son: artritis reactiva, espondiolartropatías, síndrome hemofagocitico, dermatomiositis, trastornos granulomatosos, vasculitis, entre otras (22). En coherencia con el caso reportado, se evidencia presunciones diagnósticas iniciales que llevaron a realizar estudios para descartar condiciones infecciosas como arbovirosis, sífilis, hepatitis virales, leptospirosis o presencia de hemoparásitos además de infección por virus del Epstein Barr (19). También se considera necesario el estudio de condiciones autoinmunes, de modo que se solicitaron marcadores como el factor reumatoideo, anticuerpos antinucleraes (ANA) y anticuerpos antiDNA, entre otros (23). A esto se suma el uso de estudios de tinción para biopsia de piel que no arrojaron marcadores orientativos claros, con lo que se refuerza el concepto de un diagnóstico predominantemente clínico.

Para el manejo de la paciente se recurrió al uso de esteroide oral (prednisolona $0,5 \mathrm{mg} / \mathrm{kg}$ ), con lo cual se obtuvo una respuesta significativa, reportándose disminución de las lesiones cutáneas, mejoría del dolor, disminución de la fiebre y tendencia a la disminución de transaminasas, LDH y PCR. Debido a la baja incidencia y prevalencia de la enfermedad no existen estudios prospectivos, aleatorizados doble ciego para determinar esquemas terapéuticos óptimos. La eficacia del manejo se obtiene de reportes de casos y estudios retrospectivos de pequeña escala (24). Los antiinflamatorios no esteroideos y la prednisolona son usados como agentes de primera línea; en casos de compromiso visceral se logra obtener respuesta con infusión 
intravenosa de altas dosis de metilprednisolona $(25,26)$; también se considera oportuno el uso de metotrexate en casos selectos (27). La eficacia de antagonistas de receptor de IL-1, bloqueadores del TNF, inmunoglobulinas y ciclosporina también ha sido reportado $(10,21)$.

\section{Conclusión}

El reporte de caso previamente descrito es un ejemplo de la complejidad que implica el abordaje de esta enfermedad, tanto desde el punto de vista del diagnóstico como del tratamiento. Este paciente cumplió con los criterios de Yamaguchi, lo que contribuyó a confirmar el diagnóstico. Se concluye que la enfermedad de Still del adulto no tiene manifestaciones clínicas ni pruebas de laboratorio patognomónicas, por lo que el diagnóstico continúa siendo por exclusión.

\section{Bibliografía}

1. Giacomelli R, Ruscitti P, Shoenfeld Y. A comprehensive review on adult onset Still's disease. J Autoimmun. 2018 Sep;93:24-36. DOI: 10.1016/j.jaut.2018.07.018.

2. Díez Morrondo C, Pantoja Zarza L. Aspectos actuales de la enfermedad de Still del adulto. Medicina Clínica. 2014;142(1):29-32. DOI: 10.1016/j.medcli.2013.06.001

3. Agha-Abbaslou M, Bensaci AM, Dike O, Poznansky MC, Hyat A. Adult-Onset Still's Disease: Still a Serious Health Problem (A Case Report and Literature Review). Am J Case Rep. 2017 Feb 3; 18:119-24.

4. Suárez Rodríguez BL, Díaz Padrón EG, López Suárez RI. Enfermedad de Still del adulto. Rev Cubana de Reumatolo. 2013;15(3): 204-08.

5. Jamilloux Y, Gerfaud-Valentin M, Henry T, Sève P. Treatment of adult-onset Still's disease: a review. Ther Clin Risk Manag. 2014; 11:33-43. DOI: 10.2147/TCRM.S64951

6. Ruscitti P, Giacomelli R. Pathogenesis of adult onset still's disease: current understanding and new insights. Expert Rev Clin Immunol. 2018;14(11):96576. DOI: $10.1080 / 1744666 X .2018 .1533403$

7. Bywaters EG. Still's disease in the adult. Ann Rheum Dis. 1971;30(2):121-33.

8. Mahfoudhi M, Shimi R, Turki S, Kheder A. Epidemiology and outcome of articular complications in adult onset Still's disease. Pan Afr Med J. 2015 30;22:77. DOI: 10.11604/pamj.2015.22.77.6366.

9. Gonzalez FA, Beirão P, Adrião J, Coelho ML. Adultonset Still's disease presenting as myopericarditis. BMJ Case Rep. 2014 Jun 4;2014. DOI: 10.1136/bcr2013-202754
10. Imbrechts M, Avau A, Vandenhaute J, MalengierDevlies B, Put K, Mitera T, Berghmans N, Burton O, Junius S, Liston A, de Somer L, Wouters C, Matthys P. Insufficient IL-10 Production as a Mechanism Underlying the Pathogenesis of Systemic Juvenile Idiopathic Arthritis. J Immunol. 2018 Nov 1;201(9):2654-63. DOI: $10.4049 /$ jimmunol.1800468.

11. Senthilvel E, Papadakis A, McNamara M, Adebambo I. Adult-Onset Still Disease (AOSD). J Am Board Fam Med. 2010;23(3):418-22. DOI: 10.3122/jabfm.2010.03.090157

12. Avilés ASV, Cobos REB, Rodríguez AAB, Casián Castellanos G, Balcázar Vázquez R. Enfermedad de Still de inicio en el adulto. Reporte de caso. Rev Hosp Jua Mex. 2011;78(3):187-90.

13. Jiménez-Sánchez JA, Bailón- Becerra A, Domínguez-Borgúa A. Enfermedad de Still de inicio en el adulto como causa de fiebre de origen desconocido. Med Int Mex. 2016;32(1):118-28.

14. Antoniou KM, Margaritopoulos GA, Giannarakis I, Choulaki C, Fountoulakis N, Siafakas NM, et al. Adult Onset Still's Disease: A Case Report with a Rare Clinical Manifestation and Pathophysiological Correlations. Case Rep Med. 2013;2013. DOI: $10.1155 / 2013 / 981232$

15. García-García G, Fernández-Auzmendi V, OlgadoFerrero F, Magro-Ledesma D, Sánchez Giralt S. Miopericarditis aguda como presentación de enfermedad de Still del adulto. Reumatología Clínica. 2012 ene-feb;8(1):31-3. DOI: 10.1016/j.reuma.2011.03.002

16. Panqueva U, Ramírez LA, Restrepo JF, Rondón F, Mora S, Valle R, et al. Enfermedad de Still del Adulto: Estudio de Cohorte. Rev Colomb Reumatol. 2009; 16(2):326-81. DOI: 10.1016/S0121-8123(09)70097-0

17. Mitchell PD, Viswanath A, Obi N, Littlewood A, Latimer M. A prospective study of screening for musculoskeletal pathology in the child with a limp or pseudoparalysis using erythrocyte sedimentation rate, C-reactive protein and MRI. J Child Orthop. 2018 Aug 1;12(4):398-405. DOI: 10.1302/1863-2548.12.180004.

18. Pouchot J. Enfermedad de Still del adulto. EMC - Tratado de Medicina. 2013;17(2):1-8. DOI: 10.1016/S1636-5410(13)64523-7

19. Copland A, Bending D. Foxp3 Molecular Dynamics in Treg in Juvenile Idiopathic Arthritis. Front Immunol. 2018 Oct 2; 9:2273. DOI: 10.3389/fimmu.2018.02273

20. Siddiqui MR, Khan GK, Islam MS, Ahmed KA. Adult Onset Still's Disease: A Case Report. Anwer Khan Modern Medical College Journal.2013;4(2):52-4. 
DOI: $10.3329 /$ akmmcj.v4i2.16944

21. Gopalarathinam R, Orlowsky E, Kesavalu R, Yelaminchili S. Adult Onset Still's Disease: A Review on Diagnostic Workup and Treatment Options. Case Rep Rheumatol. 2016;2016. DOI: $10.1155 / 2016 / 6502373$

22. Consolo M, Amoroso A, Vinci M. Adult-Onset Still's Disease, an improbable differential diagnosis: a case report. Clin Ter. 2014;165(2):99-102. DOI: 10.7471/CT.2014.1684.

23. Gerfaud-Valentin, M., Maucort-Boulch, D., Hot, A., Iwaz, J., Ninet, J., Durieu, I \& Sève. Adult-onset still disease: manifestations, treatment, outcome, and prognostic factors in 57 patients. Medicine. 2014;93(2):91-99. DOI: 10.1097/MD.0000000000000021

24. Bagnari V, Colina M, Ciancio G, Govoni M, Trotta F. Adult-onset Still's disease. Rheumatol Int. 2010 May;30(7):855-62. DOI: 10.1007/s00296-009-1291-y
25. Morgan J. Juvenile idiopathic arthritis at school: who understands? Lancet Child Adolesc Health. 2018 Nov;2(11):776-777. DOI: 10.1016/S23524642(18)30311-0.

26. Yoo DH. Treatment of adult-onset still's disease: up to date. Expert Rev Clin Immunol. 2017 Sep;13(9):849-866. DOI: 10.1080/1744666X.2017.1332994.

27. Junge G, Mason J, Feist E. Adult onset Still's disease-The evidence that anti-interleukin1 treatment is effective and well-tolerated (a comprehensive literature review). Semin Arthritis Rheum. 2017 Oct;47(2):295-302. DOI: 10.1016/j.semarthrit.2017.06.006.

28. Kadavath S, Efthimiou P. Adult-onset Still's disease-pathogenesis, clinical manifestations, and new treatment options. Ann Med. 2015 Feb;47(1):6-14. DOI: $10.3109 / 07853890.2014 .971052$. 
